\title{
Rough Texton based Fundus Image Retrieval
}

\author{
Krishnaveni Sadarajupalli \\ Computer Science and Engineering \\ Vasireddy Venkatadri Institute of Technology \\ Nambur, Guntur, A.P., India
}

\author{
Sudhakar Putheti \\ Computer Science and Engineering \\ Vasireddy Venkatadri Institute of Technology \\ Nambur, Guntur, A.P., India
}

\begin{abstract}
Content based image retrieval (CBIR), is a robust technique widely used in the field of image retrieval. This method uses visual contents like color, texture and shape, to search images from a large scale database of images. Among the primary image contents, texture is an important spatial feature. Texton is a statistical approach used to analyze the texture of an image. Texture-based approach proposed here can take into account the vagueness of images also while retrieving images just as an expert manually retrieves medical images.
\end{abstract}

\section{Keywords}

Content Based Medical Image Retrieval, Rough sets, Texture analysis, Texton, Rough Set Support Vector Machines, HSV.

\section{INTRODUCTION}

In our day to day life like government, commerce, academics, medicine, healthcare, crime prevention, security, defense, remote sensing, weather fore casting, architecture, media, graphic design, fashion and historical research are using images for best results. The use of images by all these fields created a demand for a pool of images. An image database is a set of stored images [1]. Image database fundamentally comprises the raw images and the information extracted from images by a computer assisted image analysis. One of the examples of image databases are the database of images of criminals, and images of items that were stolen are maintained by the police. Image database containing X-rays, scanned images used for diagnosis in the medical profession is another example of an important image database. A huge image database is generated in the fields of publishing and advertising. In historical research, image databases are created for archives in areas [1]. Retrieving an exact image from such a large database is difficult and at times impossible owing to the size of the database and also due to the complex process involved in identifying images with similar features. So, an automated system is required to minimize the time taken to identify the image and also to identify similar images form a vary large scale image database. Content Based Image Retrieval (CBIR) is a set of techniques can be used in retrieving similar images from an image database based on automatically-derived image features [2] [3] [4].

CBIR system includes two major steps named feature extraction and similarity measurement. Feature extraction includes the representation of image by a set of features. Similarity measurement is finding a distance between the query image and each image in the database using their feature vectors. First and foremost step of content based image retrieval system is Feature extraction. It is base for extracting unique information from the image. These features are also termed as signature of image. Same signature of two images is nothing but the two images are one and the same. It does not result any complication as the feature extraction of the image from the database is done offline. The improved technology and efficiency of CBIR reduces the need of human intervention. CBIR system also includes similarity comparison and can be achieved by extracting feature of every image. Extraction of feature is done based on its pixel values and devising rules for comparing images. Some CBIR systems employ extraction of features from the entire image than from restricted some regions in it. And these features are Global features. The extracted features of each and every image of database are stored in the database. After calculating the feature vectors of a query image it is then compared with the already stored feature vectors of the images of the data base. Based on the comparison results the images of the data base will be displayed whose feature vectors are having similar signatures with that of query image. The most similar images are displayed first and the least one on the last. According to distances of their feature vector.

For diagnostics and therapy in medical field, use of digital images is essential and it became a fundamental component for diagnosis [5], treatment planning [6], assessing response to treatment [7], as well as Picture Archiving and Communication Systems (PACS). Use of Content based access to medical images would helps in managing clinical data. Hence the combined use of content-based access methods and PACS have been developed. When applied to multidimensional and multimodality medical data it is proven that Medical CBIR is an established field of study. The question of image similarity has important applications in the medical domain because diagnostic decision-making has traditionally involved using evidence from a patient's data (image and non image) coupled with the physician's prior experiences of similar cases [8]. A recent study [9] has shown that manual selection of these similar cases is primarily based upon visual properties. It has been suggested that the reliance on imaging for various clinical workflows means that access to relevant stored data will allow for more informed and effective treatment [10].

Diabetic retinopathy (DR) is a vision-threatening complication that will found in people who are suffering from diabetes over a long time. The effect of DR on vision will be nullified to maximum extent if detected and diagnosed at early stages. As DR is a complication associated with retina and leads to blindness, more number of public initiatives are interested for detecting and diagnosing of retinal disorders in urban population. For detecting and diagnosing of retinal disorders use of digital fundus (retina) images is essential. Use of Digital color fundus (retina) images in a noninvasive manner makes large scale screening easier. As the use of color fundus images (CFI) involves low cost and wider reach it has been significant effort towards building screening solutions for DR [11] now a days.

Content-Based Image Retrieval can be employed to provide a clinician with instant references to archival and standardized images which are clinically closer to the image under diagnosis. This is an innovative way of utilizing the vast expert knowledge hidden in archives of previously diagnosed fundus camera images that helps an ophthalmologist in improving the performance of diagnosis. 
This paper contains : objective and ideas of the present approach in section 2. Section 3 deals with the features of real-world image texture analysis and rough sets for image processing. Section 4 consists of experimental results, accuracy tables. Section 5 concludes along with references.

\section{EXISTING SYSTEM}

In evidence-based medicine, in training ophthalmologists and in automated computer assisted diagnosis, essential requirement of the system is to retrieve the correct and close images depending on the pathologic state. And this has wide application because of its powerful functionality.

\subsection{Hue Saturation Value (HSV)}

For designing and implementation of image and video processing tasks Color image processing technique is a good resource. Usually any object can be easily discriminated and identified by colour, which is mostly used visual feature for content-based image retrieval. It is necessary to have effective color space to extract colour features of image. To define the specifications of colours, color space required. Coordinate system is used to represent the colours in a colour space. Each colour will be represented as a point in the coordinate system.

Representation of colors in terms of intensity values is called colour space model. A way of representation of colours as tuples of numbers, usually three to four values of colour components can be called as an abstract mathematical model of colour space model. this paper they are: RGB, HSV, $\mathrm{YCbCr}$ are the three different color space that are used in this paper.

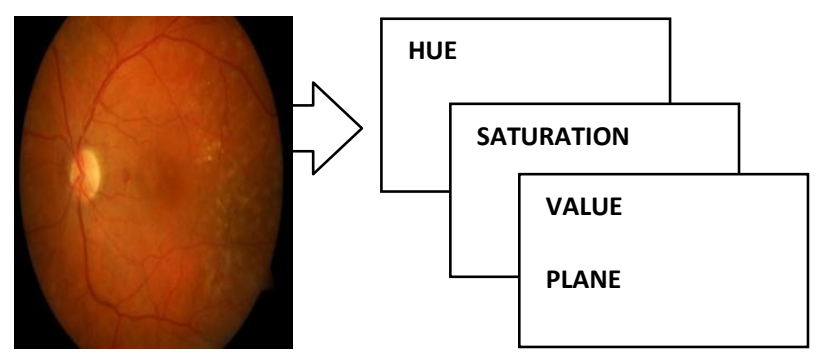

Figure 1. HSV Planes of Color Image

HSV stands for Hue, Saturation and Value is abbrivated as HSV, where Hue and Saturation defines chrominance, Value is the intensity which denotes luminance [12]. Colours can be distinguished by using Hue, percentage of white light added to the pure colour will be measured by saturation. And intensity of the light is nothing but the Value. These three dimensional representation with the use of HSV color space is termed as a hexacone, and intensity is represented by the central vertical axis [9]. Hue is defined as an angle in the range $[0,2 \pi]$ relative to the Red axis with red at angle 0 , green at $2 \pi / 3$, blue at $4 \pi / 3$ and red again at $2 \pi$. The depth or purity of the color is Saturation and will be measured as a radial distance from the central axis. The value of $\mathrm{S}$ lies between 0 and 1 . At the center it is 0 and at the outer surface it will be 1 . When $S=0$, and for the movement of higher intensity along its axis one moves from black to white by shades of gray. When $S=1$ for the movement of higher intensity along its axis one goes from black to white by purest form of color represented by its Hue rather than gray shade. The feature generation provides an approximation colour of every pixel in thresholding form. HSV color space can be used for generation of histogram.
Number of colours can be quantized into several bins to reduce the complexity involved by more number of colours.

\subsection{Multi Texton Histogram}

The study of effortless texture discrimination can serve as a model system. The roles of local texture detection and statistical computation in visual perception can be distinguished [1] with the use of model system. This can easily be explained by the local orientation differences between the elements having two texture images. The differences between the texture images will be described globally. Even if the two texture images are identical in their first-order statistics, the second-order statistics may differ greatly [13]. The first and second-order statistics have their own advantages in texture discrimination. Hence the first order statistics and second-order statistics can be combined as a single entity for texton analysis. The technique multi-texton histogram (MTH), is used for image retrieval. Based on the texton theory $[13,14]$, texture can be decomposed into elementary units like the texton classes of colors, orientation and aspect ratios, elongated blobs of specific widths and the terminators of these elongated blobs. Texture orientation needs to be detected for texton analysis in the MTH-based image retrieval scheme.

Multi Texton Histogram (MTH) is one of the methods for image retrieval which employsfeature extraction technique. Inspite of its ability to represent the image well, it has the following drawbacks. First, for image representation MTH uses only local features. Second, image representation may degrade because of some sort of missing information in the process of pixel pair detection using texton. The MTCD extracts color, texture and shape features simultaneously using texton. And image representations is calculated globally using Gray Level Co-occurrence Matrix (GLCM). Using Canberra image similarity will be calculated and using precision and recall performance of MTCD is measured.

\subsection{Rough Set}

Rough set theory is a new mathematical tool for vague or imperfect data analysis. In areas like banking, medicine, decision support, engineering environment the idea of rough set theory has its own significance [15]. Rough set theory is developed as such any object in universe has some information associated with it. Objects having the same information are similar. Rough set theory is developed based on the relation generated based on similarity among objects. Hence an elementary set is defined as a set of similar objects constitutes a minute part. With this knowledge about elementary set a crisp (precise) set can be defined as a union of some elementary sets, also a set which is not crisp (precise) is called rough (imprecise, vague) set. Every rough set has certain boundary line elements, the objects which cannot be certainly classified with the available information associated with them will fall in that boundary line. Hence the rough sets cannot be characterized on the basis of information about their elements as if, which is the case with precise set. Hence every rough set will be associated with a pair of precise sets called lower approximation set and upper approximation set. The lower approximation set consists of all objects which are certainly classified with available information and completely belongs to that set. The upper approximation set consists of all objects which may possibly belongs to that set. The elements or objects which does not belong to lower and upper approximation sets forms the boundary region of the rough set [16]. 
A decision table is used for analysis of rough set based data. Columns of this decision table consist of attributes. Rows by of the decision table consist of object of interest. And the attribute values will be the entries of the table. Two disjoint groups of attributes called condition and decision attributes forms a decision table. Every row of decision table has its own decision rule in terms of conditions. The rule of decision is certain if it is able to determine the decision in terms of conditions. And the rule of decision is not certain if it is not able to determine the decision in terms of condition. Hence certain decision rule refers to lower approximation set and uncertain decision rule refers to boundary region.

Certainty, coverage coefficient are two conditional probabilities that associates decision rule. Among these two coefficients certainty provides information regarding the conditional probability whether a particular object belongs to the decision class which is specified by the decision rule and satisfies conditions of the rule. The coverage coefficient gives information about the conditional probability reasons of a given decision. Both certainty and coverage coefficients satisfy Bayes' theorem and helps into interpretation of Bayes' theorem, and thus conclusions will be drawn from data in a new method. Rudiments of the theory, with basic concepts will be explained in this paper by means of an example of churn modeling. But more advanced extensions of the theory is required in real life applications, however which is not discussed in present paper. Rough set theory is having similarities with other theories dealing with imperfect knowledge such as fuzzy sets, Bayesian inference,evidence theory, and others.

\section{PROPOSED SYSTEM}

In this proposed system rough texton approach is used to extract the texture and rough set-support vector machine for relevance feedback.

\subsection{Rough Texton}

As per Julesz [17] description a texton is a pattern which is shared by an image as a common property. Textures will be formed only if the adjacent elements lie within the neighborhood. Texton Image has the discrimination power of color, texture and shape features. Based on the Julez's [17] textons theory the texton co-occurrence matrices (TCM) algorithm, can describe the spatial correlation of textons for image retrieval. With the use of limited number of selected pixels the algorithm computes different features. Retrieval efficiency in CBIR will be increased by calculating texton using Rough texture spectrum and color features are calculated using HSV color space. Compared to RGB, HSV has its advantages. When turned as a shape descriptor, texton deals directly with natural images and any segmentation or object extraction preprocessing stage will not be done. As a texture descriptor, good retrieval accuracy can be achieved especially for directional textures.

The texton can be inclined with the use of critical distances between texture elements. Texture element size determines the critical distance. Texture can be resolved into minute units, like orientation, Texton classes of colors, elongated blobs of specific width, aspect ratios and terminators of elongated blobs. If texture elements are expanded to a large extent in one orientation discrimination reduces. If the elongated elements are not jittered in orientation texture gradients increase at boundaries. Hence by using a sub image of size $3 \times 3$ a texton gradient can be obtained. Here 12 textons of $3 \times 3$ grids are proposed as shown in figure 2 . Even the co-occurrence probability of same valued pixels in $3 \times 3$ grids is smaller than that of $2 \times 2$ grid, the textons developed using $2 \times 2$ grid may not give complete information regarding direction. The computational complexity for using the overlapped components of 12 textons is also less to obtain final texton image. The 12 textons of $3 \times 3$ grid [17] is used in the proposed method.

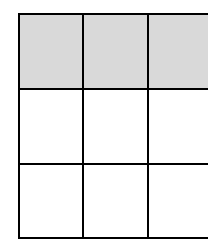

(a)

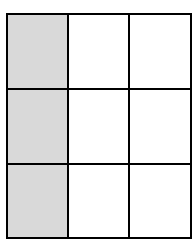

(d)

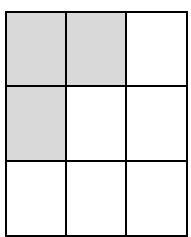

(g)

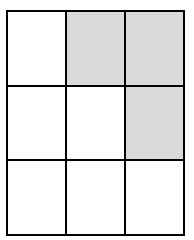

(j)

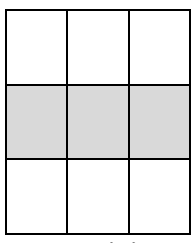

(b)

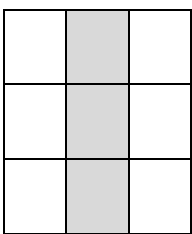

(e)

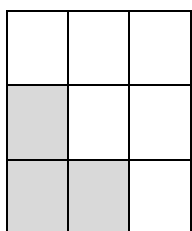

(h)

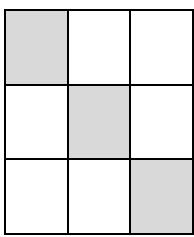

(k)

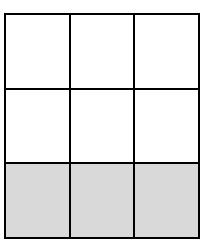

(c)

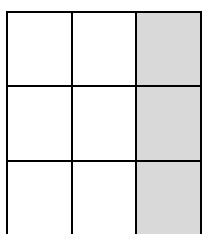

(f)

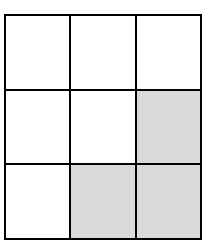

(i)

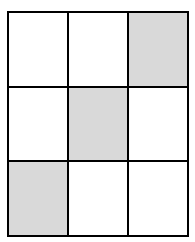

(I)
Figure 2. 12 types of $3 \times 3$ textons

Determination of texture property can be done based on the values of Texture Unit and Texture Unit Number of an image plane [17]. A $3 \times 3$ pixel square will be constructed to define a basic unit of the method. $3 \times 3$ pixel square consists of a central pixel surrounded by eight neighbors. The square image has the information regarding local texture in all directions of the central pixel.

Extraction of information regarding local texture of the surrounding pixels is the main objective. The size of the neighborhood is $3 \times 3$ pixels in present case. The nine pixels of this $3 \times 3$ square pattern of the image is denoted $\mathrm{V}$, where $\mathrm{V}$ consists of nine elements as $\mathrm{V}=\{\mathrm{V} 0, \mathrm{~V} 1, \mathrm{~V} 2 \ldots \mathrm{V} 8\}$, where V0 corresponds to the central pixel's intensity and Vi $(1 \leq i \leq 8)$ corresponds to intensity surrounding pixel. Texture Unit (TU) is nothing but the unit which best suits for the local texture feature of a given pixel and its neighborhoods, in all directions of a square raster. Texture Unit (TU) as in [17], will be given by $T U=\{E 1, E 2, \ldots, E 8\}$, where: 


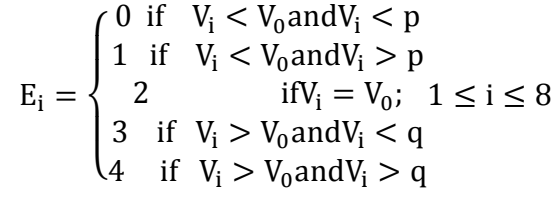

where pre defined or user defined values will be assigned to $p$ and q. And element Ei occupies the same position as occupied by pixel $i$.

\begin{tabular}{|c|c|c|}
\hline 121 & 190 & 67 \\
\hline 225 & 180 & 252 \\
\hline 180 & 170 & 201 \\
\hline
\end{tabular}

(a)

\begin{tabular}{|c|c|c|}
\hline 1 & 3 & 0 \\
\hline 4 & & 4 \\
\hline 2 & 1 & 3 \\
\hline
\end{tabular}

(b)
Figure 3(a) Hue levels of an image part. (b) Texture Unit associated to the central pixel.

The total number of TU is $390625\left(5^{8}\right)$. That can be obtained that each element of the TU can have any one of three possible values $0,1,2,3$ or 4 . TU can be labeled and can be ordered in different ways; each TU will be labeled as a 5-base number, named Texture Unit Number, $\mathrm{N}_{\mathrm{TU}}$ will be calculated by suing the formula:

$$
\mathrm{N}_{\mathrm{TU}}=\sum_{\mathrm{i}=1}^{8} \mathrm{E}_{\mathrm{i}} \cdot 5^{\frac{\mathrm{i}-1}{2}}
$$

where $\mathrm{i}$ denotes position of Texture Unit box, and Ei corresponds to value of the box $(0,1,2,3$ or 4$)$. The 8 elements can be ordered differently. As shown in figure 4 where the elements are ordered in clock wise direction, the first element can have eight possible positions, from ' $a$ ' to ' $h$ 'in clockwise direction and then the 16777216 texture units can be labeled by the abode formula under eight different ordering ways (from a to $h$ ).

\begin{tabular}{|c|c|c|}
\hline $\mathrm{a}$ & $\mathrm{b}$ & $\mathrm{c}$ \\
\hline $\mathrm{h}$ & & $\mathrm{d}$ \\
\hline $\mathrm{g}$ & $\mathrm{f}$ & $\mathrm{e}$ \\
\hline
\end{tabular}

\section{Figure 4 Texture Unit Ordering}

A more detail study of texture unit indicates that the absent TU's involve 2's in their texture unit. This is the case when neighbors and central pixels have the same values. If there is a lack of 2's then TU will take only $0,1,3$ and 4 which means that the possible real number of different textures are $4^{8}$ instead of $5^{8}$, that is 65536 and 390625 the spectrum will be never totally covered, thus the power of the method is misused. It impacts on texture Unit number also. To overcome this, Rough texture is used in the proposed method.

Rough Texton is a way to describe the texture property in a way better than the Texton since Rough texton uses the Rough texture unit (RTU) instead of texture unit (TU). By including rough set [16] concept to the texton will generates all ranges of the values including ' 2 ' in the RTU. Then, it helps to generates different textures are $5^{8}$ instead of $4^{8}$. It covers total spectrum which is not possible by texton only means without rough set concept. Using Rough Set techniques provide a more flexible way of assigning values to the $T U$ boxes $E_{i}$.
It can also be mentioned that rough set theory [16], in other side to fuzzy set theory, evidently distinguishes two important concepts like vagueness and uncertainty, these two concepts are often confused. Vagueness of a set can be defined by approximations. uncertainty relates to the elements of a set, such that the properties of elements are to be explained by the rough membership function.

\subsection{Rough Sets - Support Vector Machines approaches}

Based on guaranteed risk bounds of statistical theory of learning a general algorithm called Support Vector Machines (SVMs) is developed. SVM has its significance in process of image processing and pattern recognition. Also helpful while detecting the micro-calcification (MC) clusters in digital mammograms and other medical diagnostics. And because of advantages of its own it has been effectively used as effective computational tool. A Rough set support vector machines (RS-SVMs) is proposed by Gexianget al. [16, 18] which includes the advantages of both Rough Set and SVMs. This RS-SVMs can be used to recognize radar emitter signals. The performance of SVMs can be improved with the use of Rough set. Experimental results have shown that the RS-SVMs has lower recognition error rates than SVMs. In particular case where the training samples are less RS-SVMs are more efficient SVMs.

Lingras and Butz $[16,19]$ described interpretation of binary classification with SVMs using rough sets. Also described how to reduce the storage requirements of the 1-v-1 approach in the operational phase using rough set theory. Their techniques provided better semantic interpretations of the classification process. Experiments shown a synthetic dataset supported the theoretical conclusions. For soft margin classifiers in solving medical imaging problems especially a multi-class classification system for medical images the presented work is useful $[16,20]$.

Yun et al. $[16,21]$ have used a rough-support vector machine integration to develope the Improved Support Vector Machine (ISVM) algorithm. This helps to classify digital mammography images, where rough sets are applied to reduce the original feature sets and the support vector machine is used classify the reduced information. The ISVM classifier can get $96.56 \%$ accuracy which is shown by the experimental results and which is higher than $92.94 \%$ which is the case using SVM, and the error recognition rates are close to $100 \%$.

\subsection{CBMIR}

For content based medical image retrieval system the proposed texture descriptor is developed, which is used to retrieve most similar medical images for the given query image as shown in the figure 5 . There are two major steps in CBMIR, namely feature extraction and similarity measurement. A set of features are generated to represent the content of each image in feature extraction. similarity measurement gives the similarity distance between the query image and each image in the database which will be computed by their feature vectors.

Using the description of the texture the texture feature extracted will be considered for retrieving the medical images from the large collection of medical database. The following are the various steps involved in the CBIR system:

i) For the gray scale images that are stored in the data base Rough Texton images are to be calculated. 
ii) Cooler Quantization is performed in HSV color space for the color image.

iii) For the HSV Planes Rough Texton images are calculated.

iv) The texture feature is extracted from data base, by texture descriptor method and texture features are incorporated in the database.

v) With a known data set and with the use of Rough Set Support Vector Machine the image retrieval system is trained.

vi) Using the proposed texture descriptor as followed in the steps one, two and three, the texture feature is extracted to match the given image.

vii) The similarity distance of each image in the database and the query image is computed which is nothing but similarity measurement.

viii) Based on the similarity distance the images are arranged according to their feature vector to the query image.

ix) The images having similar signatures from the data base will be retrieved and displayed.

x) Most appropriate images from the data base will be retrieved by the trained image retrieval system.

xi) Feedback of users helps to improve system to extract most appropriate images.

\section{EXPERIMENTAL RESULTS AND DISCUSSION}

In this section, the experiments that have been carried out to test the efficiency and effectiveness of proposed framework are presented. The performance of the proposed system is tested on large medical image database which consists of images Retinal images in terms of Precision and Recall [22].

The experimental results retinal images are shown in Table 1. Figure 7 shows the sample output of a query image as shown in figure 6 using the proposed retrieval system from texture (rough texton) and classifier (SVM) properties.

The performance measures used for comparisons are
i) Mean Average Precision (MAP)
ii) R-Precision
iii) Precision@10 and
iv) Recall@ 10

Table 1 Performance Comparison for Retinal Images

\begin{tabular}{|c|c|c|c|c|}
\hline $\begin{array}{c}\text { Method } \\
\text { Performance }\end{array}$ & $\begin{array}{c}\text { MAP } \\
(\mathbf{\%})\end{array}$ & $\begin{array}{c}\text { R-Prec } \\
(\mathbf{\%})\end{array}$ & $\begin{array}{c}\text { P@ 10 } \\
(\mathbf{\%})\end{array}$ & $\begin{array}{c}\text { R@ 10 } \\
(\mathbf{\%})\end{array}$ \\
\hline MTH & 70.32 & 76.57 & 85.37 & 76.65 \\
\hline Rough Texton & 75.46 & 79.84 & 89.01 & 79.49 \\
\hline $\begin{array}{c}\text { Rough Texton } \\
\text { and RS-SVM }\end{array}$ & 86.05 & 88.14 & 97.03 & 90.06 \\
\hline
\end{tabular}

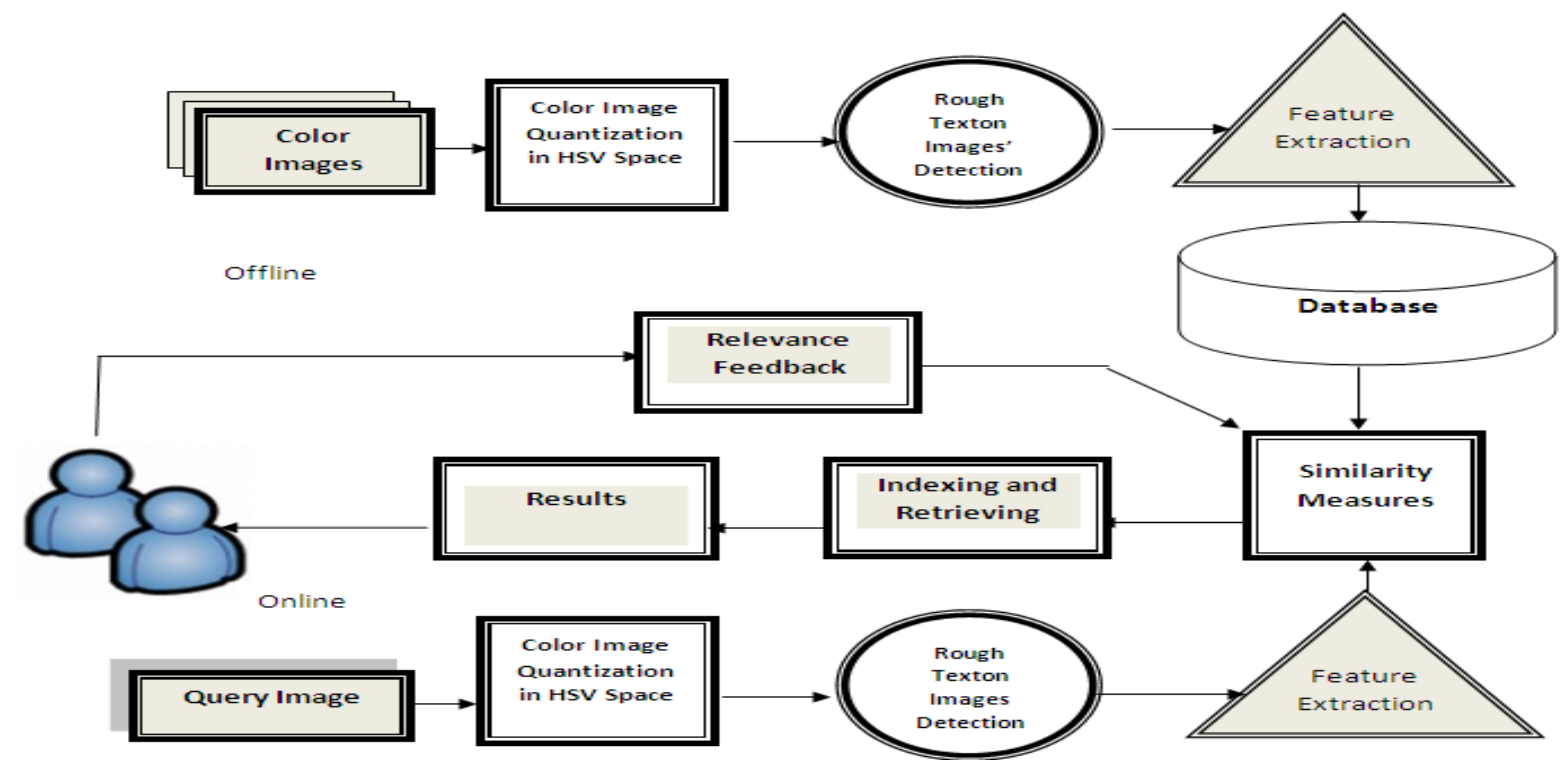

Figure 5 Content Based Medical Image Retrieval System Using Texture Descriptors

From the Table 1, it was found that the proposed retrieval system outperforms. The proposed retrieval system has the mean average precision of $86 \%$. The precision for retrieving top 10 images (P@10) is nearly $98 \%$ in the proposed retrieval system. This high retrieval performance is due to the fact that the gap between the high level semantics and low level visual features has been reduced in the proposed retrieval system. Figure 8 gives the comparison chart of the various performance measures. Figure 9 gives the precision-recall graph in which the precision is recorded on the different levels of recall. From this graph also it was found that the proposed retrieval system accuracy is substantially improved 


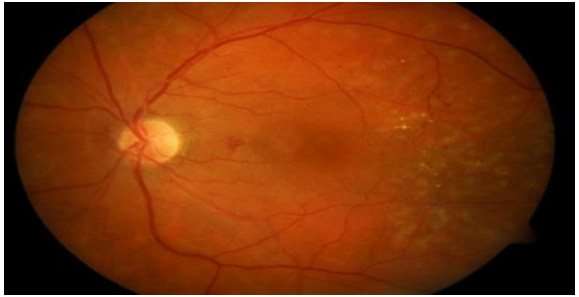

Figure 6. Query Retinal Image

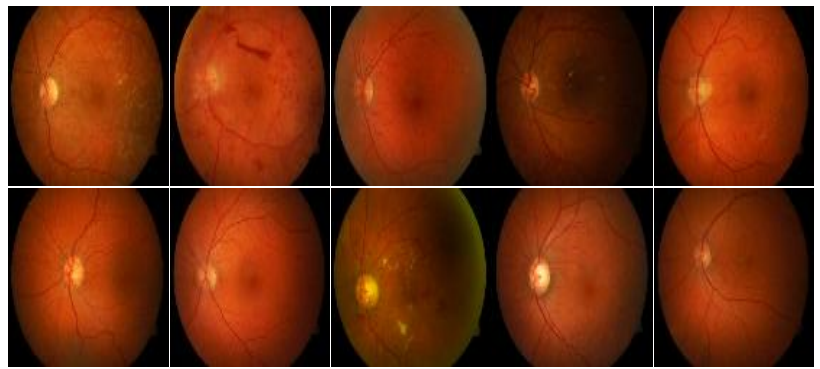

Figure 7 Retrieved retinal images from the proposed method

$\square$ MTH $\square$ Rough Texton $\quad$ Rough Texton and RS-SVM

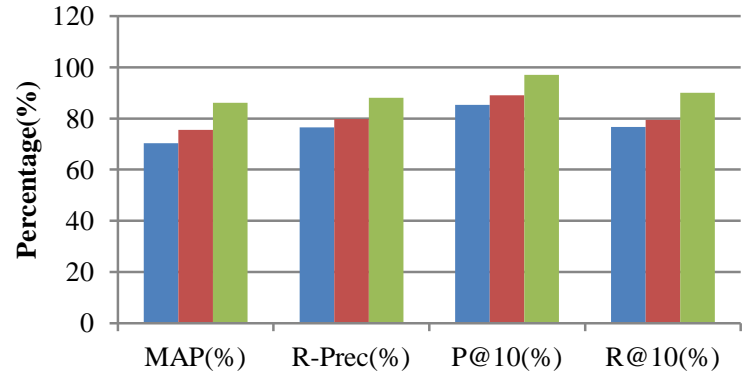

Figure 8 The comparison chart of the various performance measures.

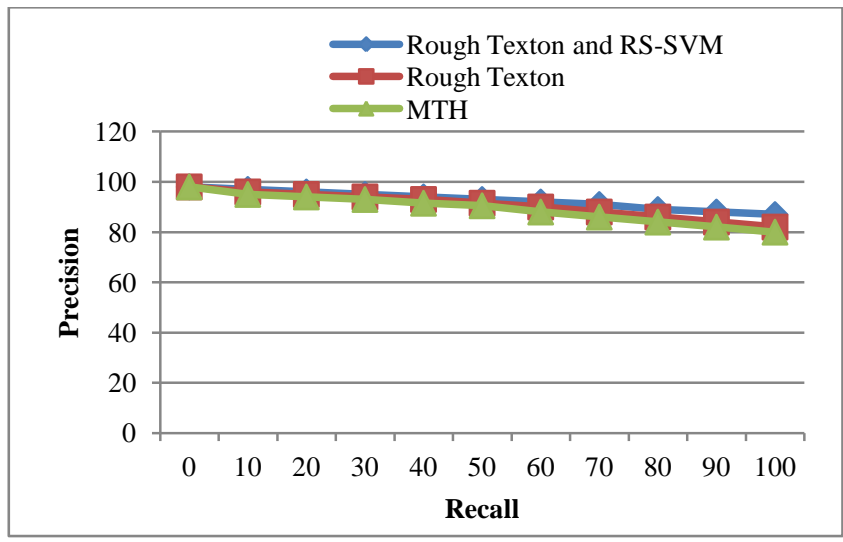

Figure 9 The Precision-Recall Graph

\section{CONCLUSION}

We propose a new method, namely Rough Texton (RT), which explains image texture for image retrieval. RT is efficient to give information regarding both color and texture based on Rough Set. Proposed method is an advanced method to retrieve an image with better texture by Rough texture unit than texture unit. It is useful to the end user for improving success rate of the image retrieval.

\section{REFERENCES}

[1] Chi Kuo Chang, "Image Information Systems," Proc. of IEEE Pattern Recognition, vol. 73, no 4, pp.754 - 766, April 1985.

[2] S. Gerard, C. Buckely, "Term-Weighting Approaches in Automatic Text Retrieval," Information Processing and Management, vol. 24, no.5, pp. 513-523, Jan. 1988.

[3] Y. Chen, J. Wang, "Image Categorization by Learning and Reasoning with Regions," Journal of Machine Learning Research, vol. 5, pp. 913-939, May 2004.

[4] F. Long, H. Zhang, H. Dagan, and D. Feng, "Fundamentals of content based image retrieval," in D. Feng, W. Siu, H. Zhang (Eds.): "Multimedia Information Retrieval and Management. Technological Fundamentals and Applications," Multimedia Signal Processing Book, Chapter 1, Springer-Verlag, Berlin Heidelberg New York, 2003, pp.1-26.

[5] Doi K: Computer-aided diagnosis in medical imaging: Historical review, current status and future potential. Comput Med Imaging Graph 31(4-5):198-211, 2007

[6] Zaidi H, Vees H, Wissmeyer M: Molecular PET/CT imagingguided radiation therapy treatment planning. Acad Radiol 16(9):1108-33, 2009

[7] Marcus C, Ladam-Marcus V, Cucu C, Bouché O, Lucas $\mathrm{L}$, Hoeffel $\mathrm{C}$ : Imaging techniques to evaluate the response to treatment in oncology: Current standards and perspectives. Crit Rev Oncol/ Hematol 72(3):217-38, 2009

[8] . Holt A, Bichindaritz I, Schmidt R, Perner P: Medical applications in case-based reasoning. Knowl Eng Rev 20(03):289-92, 2005

[9] Sedghi S, Sanderson M, Clough P: How do health care professionals select medical images they need? ASLIB Proc 64(4):437- 56, 2012

[10] Haux R: Health information systems-Past, present, future. Int J Med Inform 75(3-4):268-81, 2006

[11] J. Cuadros and G. Bresnick. EyePACS: An Adaptable Telemedicine System for Diabetic Retinopathy Screening. Journal of Diabetes Science and Technology, 3(3):509-516, 2009.

[12] S. Manimala and K. Hemachandran, "Performance analysis of Color Spaces in Image Retrieval", Assam University Journal of science \& Technology, Vol. 7, Number II 94-104, 2011.

[13] B. Julesz, Textons, the elements of texture perception and their interactions, Nature 290 (5802) (1981) 91-97.

[14] B. Julesz, Texton gradients: the texton theory revisited, Biological Cybernetics 54 (1986) 245-251.

[15] Aboul Ella Hassanien, Ajith Abraham, James F. Peters, Gerald Schaefer, Christopher Henry, "Rough Sets and Near Sets in Medical Imaging: A Review", IEEE trans. on information technology in biomedicine, 2009.

[16] Z. Pawlak, "Classification of objects by means of attributes," Polish Academy of Sciences, vol. 429, 1981.

[17] S Putheti, SR Edara, SA Edara, "CBIR using Texels of colour Fuzzy Textons", Hybrid Intelligent Systems 
(HIS), 2012 12th International Conference on, 461-467, 2012.

[18] G. Zhang, Z. Cao, and Y. Gu, "A hybrid classifier based on rough set theory and support vector machines," Lecture Notes in Computer Science, vol. 3613, pp. 306333, 2005.

[19] P. Lingras and C. Butz, "Rough set based 1-v-1 and 1-v-r approaches to support vector machine multiclassification," Information Sciences:an International Journal, vol. 177, no. 18, pp. 3782-3798, 2007.
[20] B. Qiu, C. Xu, and Q. Tian, "An automatic classification system applied in medical images," in IEEE Int. Conf. on Multimedia and Expo, 2006, pp. 1045-1048.

[21] Y. Jiang, Z. Li, L. Zhang, and P. Sun, "An improved SVM classifier for medical image classification," in Int. Conf. on Rough Sets and Emerging Intelligent Systems Paradigms, LNAI, vol. 4585, 2007, pp. 764-773.

[22] Olson, David L.; and Delen, Dursun (2008); Advanced Data Mining Techniques, Springer, 1st edition (February 1, 2008), page 138, ISBN 3-540-76916-1 